\title{
A SIMPLE SET WHICH IS NOT EFFECTIVELY SIMPLE
}

\author{
GERALD E. SACKS ${ }^{1}$
}

For each $e$, let $f_{e}$ be the partial recursive function

$$
U\left(\mu y T_{1}(e, n, y)\right),
$$

and let $W_{e}$ be the range of $f_{e}$. Then $W_{0}, W_{1}, W_{2}, \cdots$ is the Kleene enumeration of the recursively enumerable sets. Post [5] calls a recursively enumerable set simple if its complement is infinite but does not contain any infinite, recursively enumerable set. Raymond Smullyan calls a recursively enumerable set $W$ effectively simple if its complement is infinite, and if there is a partial recursive function $f$ such that for each $e$, if $W_{e}$ is contained in the complement of $W$, then $f(e)$ is defined and is greater than the cardinality of $W_{e .}{ }^{2}$ Clearly, an effectively simple set is simple. The simple set $S$ constructed by Post in [5] is effectively simple. This latter is no accident. In fact it is not unreasonable to claim that any direct attack on the problem of constructing a simple set must result in an effectively simple set. Our purpose here is to obtain a simple set which is not effectively simple. We will make strong use of the recursion theorem of Kleene [2]; however, we will use it in the informal manner of Myhill [4]. Our notation is that of [2].

We introduce a recursive function $E$ :

$$
\begin{aligned}
E(0) & =\mu x T_{1}\left((x)_{0},(x)_{1},(x)_{2}\right) ; \\
E(s+1) & =\mu x\left[x>E(s) \& T_{1}\left((x)_{0},(x)_{1},(x)_{2}\right)\right] .
\end{aligned}
$$

We will need $E$ to simultaneously enumerate all the recursively enumerable sets in a fashion suitable for the proving of our theorem. It is a peculiarity of our proof that we cannot rely merely on the usual properties associated with any standard enumeration of the recursively enumerable sets; instead, we are forced to specify a particular enumeration. For each $e$ and $s$ we define a finite set $W_{e}^{s}$ : for each $m, m \in W_{e}^{s}$ if and only if for some $i \leqq s$,

$$
m=U\left((E(i))_{2}\right) \& e=(E(i))_{0} .
$$

Then for each $e, W_{e}^{0} \subseteq W_{e}^{1} \subseteq W_{e}^{2} \subseteq \cdots$, and $W_{e}=U\left\{W_{e}^{s} \mid s \geqq 0\right\}$. We

Presented to the Society January 24, 1963; received by the editors October 25, 1962.

${ }^{1}$ The preparation of this paper was partially supported by N.S.F. Grant GP-124.

${ }^{2}$ Smullyan actually requires that $f$ be recursive, but it is easy to show the two definitions equivalent. 
say $s$ defines $f_{e}(n)$ if $e=(E(s))_{0}$ and $n=(E(s))_{1}$. If $s$ defines $f_{e}(n)$, then $f_{0}(n)=U\left((E(s))_{2}\right)$. For each $e$ and $n$, let

$$
S(e, n) \simeq \mu s \quad\left(s \text { defines } f_{e}(n)\right) .
$$

$S$ is partial recursive, and $S(e, n)$ is defined if and only if $f_{\theta}(n)$ is defined.

Let 0 denote the empty set. It is clear there exists a recursive function $g$ such that for each $e, i$ and $z$, we have

$$
\begin{aligned}
& W_{o(e, i, z)} \\
& \quad=\left\{\begin{array}{l}
\left\{2^{i} \cdot 3^{t} \mid E(S(e, z))<t \leqq E(S(e, z))+f_{\odot}(z)\right\} \\
0 \quad \text { otherwise. }
\end{array} \text { if } f_{e}(z)\right. \text { is defined, }
\end{aligned}
$$

The recursion theorem tells us that there exists a recursive function $z$ such that for each $e$ and $i$, we have

$$
\begin{aligned}
W_{z(e, i)} & =W_{o(e, i, z(e, i))} \\
= & \begin{cases}\left\{2^{i} \cdot 3^{t} \mid E(S(e, z(e, i)))<t \leqq E(S(e, z(e, i)))+f_{e}(z(e, i))\right\} & \text { if } f_{e}(z(e, i)) \text { is defined, } \\
0 & \text { otherwise. }\end{cases}
\end{aligned}
$$

We note some properties of $z$ :

(1) if $f_{e}(z(e, i))$ is defined, then $f_{\theta}(z(e, i))$ is equal to the cardinality of $W_{z(e, i)}$;

(2) if $i \neq j$, then $W_{z(e, i)} \cap W_{z(e, j)}=0$;

(3) if $f_{e}(z(e, i))$ is defined, then for all $n, W_{n}^{S(e, z(e, i))} \cap W_{z(e, i)}=0$;

(4) if $i \neq j$ and both $f_{e}(z(e, i))$ and $f_{e}(z(e, j))$ are defined, then $z(e, i) \neq z(e, j)$.

To prove (3), let $s=S(e, z(e, i))$ and let $m \in W_{n}^{s} \cap W_{z(e, i)}$. Then $m \leqq E(s)$, since $m=U\left((E(i))_{2}\right)$ for some $i \leqq s$, and since $E$ is an increasing function. (Recall that $U(x) \leqq x$ for all $x$.) But $m>E(s)$, since $m=2^{i} \cdot 3^{t}$ for some $t>E(s)$.

THEOREM 1. There exists a simple set which is not effectively simple.

Proof. We will define a sequence $A, B, Q_{0}, Q_{1}, Q_{2}, \cdots$ of simultaneously recursively enumerable sets. $A$ will be simple, but not effectively simple. $B$ will be such that if $e \in B$, then $W_{e} \cap A \neq 0$. We will see to it that if $W_{e}$ is infinite, then $e \in B$. Each $Q_{e}$ will be finite and will contain a set that will serve as a witness to the fact that $f_{0}$ does not effectively bound the cardinalities of the finite subsets of the complement of $A$.

Stage $s=0$. We set $A^{0}=B^{0}=Q_{i}^{0}=0$ for all $i$. 
Stage $s>0$. Let $e=(E(s))_{0}$ and $n=(E(s))_{1}$. Thus $s$ defines $f_{e}(n)$. We perform the following two operations in the indicated order:

(a) We set $Q_{j}^{s}=Q_{j}^{s-1}$ for all $j \neq e$. If there is no $i$ such that $i \leqq e$ and $n=z(e, i)$, we set $Q_{e}^{s}=Q_{e}^{s-1}$. If there is such an $i$, then by (4) it is unique. In addition, $S(e, z(e, i))$ is defined and

$$
W_{z(e, i)}=\left\{2^{i \cdot 3^{t}} \mid E(S(e, z(e, i)))<t \leqq E(S(e, z(e, i)))+f_{\bullet}(z(e, i))\right\} .
$$

We set $Q_{e}^{s}=Q_{e}^{s-1} \cup W_{s(e, i)}$.

(b) If $e \in B^{o-1}$ or if there is no $m$ such that

$$
m \in W_{\bullet}^{*} \&(j)_{j \leq e}\left(m \notin Q_{j}^{\prime}\right),
$$

then we set $B^{s}=B^{s-1}$ and $A^{s}=A^{s-1}$. If $e \notin B^{s-1}$ and there is an $m$ with the above property, let $i$ be the least one. We set $B^{s}=B^{s-1} \cup\{e\}$ and $A^{*}=A^{s-1} \cup\{i\}$.

Let $A=\bigcup\left\{A^{s} \mid s \geqq 0\right\}$ and $B=\bigcup\left\{B^{s} \mid s \geqq 0\right\}$. Since $E$ and $z$ are recursive, it follows $A$ is recursively enumerable. For each $e$, let $Q_{e}=U\left\{Q_{e}^{s} \mid s \geqq 0\right\} . Q_{e}$ is finite; in fact,

$$
Q_{\odot}=U\left\{W_{z(e, i)} \mid i \leqq e\right\},
$$

since $Q_{e}^{s-1} \neq Q_{e}^{s}=Q_{e}^{s-1} \cup W_{z(e, i)}$ if and only if $i \leqq e$ and $s=S(e, z(e, i))$.

Lемма 1. If $W_{e}$ is infinite, then $A \cap W_{e} \neq 0$.

Proof. We know $Q_{j}$ is finite for every $j$. Let $m$ be a member of $W^{*}$ which is greater than every member of $Q_{j}$ for all $j \leqq e$. Let $s$ be such that $m \in W_{e}^{s}$. First we suppose $e \in B^{s-1}$. Then there must be a $t<s$ such that $e \notin B^{t-1}$ and $e \in B^{t}$. At stage $t$ we must have performed operation (b) in such a manner that $B^{t}=B^{t-1} \cup\{e\}$ and $A^{t}=A^{t-1}$ $\cup\{i\}$, where $i \in W_{e}^{s}$. Now we suppose $e \notin B^{s-1}$. We have

$$
m \in W_{e}^{*} \&(j)_{j \leq e}\left(m \notin Q_{j}^{:}\right) \text {. }
$$

But then operation (b) at stage $s$ forces us to put a member of $W_{e}^{s}$ in $A^{*}$.

LEMMA 2. If $m \in W_{e}^{s}-Q_{j}^{s}$, then $m \notin Q_{j}$.

Proof. Suppose for the sake of a reductio ad absurdum that $m \in W_{e}^{s}-Q_{e}^{s}$ and $m \in Q_{j}$. Since $Q_{j}=U\left\{W_{z(j, i)} \mid i \leqq j\right\}$, there must be an $i \leqq j$ such that $m \in W_{z(j, i)}$. Since $W_{z(j, i)}$ is nonempty, $f_{j}(z(j, i))$ is defined. Let $t=S(j, z(j, i))$. Then $t$ defines $f_{j}(z(j, i))$, and consequently,

$$
Q_{j}^{t}=Q_{j}^{t-1} \cup W_{z(j, i)}
$$


since $i \leqq j$. Since $m \notin Q_{j}^{s}$, we must have $s<t$. Since $m \in W_{e}^{s}$, we have

$$
m \in W_{e}^{t} \cap W_{z(j, i)} \neq 0 .
$$

But this last contradicts (3).

LEMмA 3. If $m \in Q_{i} \cap A$, then there exists an $s$ and an $e$ such that $(E(s))_{0}=e<i$ and $\{e\}=B^{s}-B^{s-1}$ and $\{m\}=A^{s}-A^{s-1}$.

Proof. Since $m \in A$, there is an such that $\{m\}=A^{s}-A^{s-1}$. Let $e=(E(s))_{0}$. Since $A^{s} \neq A^{s-1}$, we must have $\{e\}=B^{s}-B^{s-1}$. In addition,

$$
m \in W_{e}^{*} \&(j)_{j \leqq e}\left(m \notin Q_{j}^{8}\right) .
$$

It follows from Lemma 2 that $(j)_{j \leqq e}\left(m \in Q_{j}\right)$. But then $e<i$, since $m \in Q_{i}$.

LEMma 4. The set $Q_{i} \cap A$ has at most $i$ members.

Proof. Suppose $m$ and $n$ are distinct members of $Q_{i} \cap A$. Lemma 3 guarantees the existence of $s(m), e(m), s(n)$ and $e(n)$ with properties as stated in the conclusion of Lemma 3. Thus

$$
\{m\}=A^{s(m)}-A^{s(m)-1} \&\{n\}=A^{s(n)}-A^{s(n)-1} .
$$

Since $m \neq n$, it follows $s(m) \neq s(n)$. But then $e(m) \neq e(n)$, since

$$
\{e(m)\}=B^{s(m)}-B^{s(m)-1} \&\{e(n)\}=B^{s(n)}-B^{s(n)-1} \text {. }
$$

We also know from Lemma 3 that $e(m)<i$ and $e(n)<i$. Thus we can map the set $Q_{i} \cap A$ in a one-to-one fashion into the set $\{e \mid e<i\}$.

Lemma 5. For each e, there is a $z$ such that $W_{z}$ is contained in the complement of $A$ and such that either $f_{e}(z)$ is undefined or $f_{e}(z)$ is not greater than the cardinality of $W_{\mathbf{z}}$.

Proof. Fix $e$. We show that some member of the sequence, $z(e, 0)$, $z(e, 1), \cdots, z(e, e)$ serves as the desired $z$. Suppose there is an $i \leqq e$ such that $f_{e}(z(e, i))$ is undefined. Then $W_{z(e, i)}=0$, and the lemma is proved. Suppose then that $f_{e}(z(e, i))$ is defined for all $i \leqq e$. By (1), $f_{e}(z(e, i))$ is not greater than the cardinality of $W_{z(e, i)}$ for any $i \leqq e$. Thus it suffices to find an $i \leqq e$ such that $W_{z(e, i)} \cap A=0$. The sets,

$$
W_{z(e, 0)}, W_{z(e, 1)}, \cdots, W_{z(e, e)},
$$

are nonempty and disjoint. If each of them has a member in $A$, then their union has at least $e+1$ members in $A$. But their union is $Q_{e}$, and Lemma 4 tells us that $Q_{e}$ has at most $e$ members in $A$.

It follows from Lemma 5 that $A$ is not effectively simple. It also 
follows from Lemma 5 that the complement of $A$ is infinite, since otherwise, the constant function

$$
f(n)=1+\text { cardinality of the complement of } A
$$

would constitute a counterexample to Lemma 5. Finally, by Lemma $1, A$ is simple.

Post [5] calls a recursively enumerable set $W$ hyper-simple if its complement is infinite, and if there does not exist a recursively enumerable sequence of disjoint, finite sets, each one of which contains a member of the complement of $W$. It can be shown with the help of Lemma 4 that $A$ is not hyper-simple.

The proof of Theorem 1 above is, as far as we know, the first proof in recursion theory to make simultaneous use of the recursion theorem and the priority method of Friedberg [1] and Muchnik [3]. The priority method was needed to resolve the inevitable conflict between putting elements in $A$ as required by Lemma 1 and keeping them out of $A$ as required by Lemma 4. Thus in operation (b), we are not allowed to take $m$ from $W_{e}^{s}$ and add it to $A^{s}$ if for some $j \leqq e, m \in Q_{j}^{s}$. The recursion theorem was needed to prove that our system of priorities does eventually resolve all conflicts happily; in particular, the recursion theorem made possible the proof of Lemma 2.

\section{REFERENCES}

1. R. M. Friedberg, Two recursively enumerable sets of incomparable degrees of unsolvability, Proc. Nat. Acad. Sci. U. S. A. 43 (1957), 236-238.

2. S. C. Kleene, Introduction to metamathematics, Van Nostrand, New York, 1952.

3. A. A. Muchnik, Negative answer to the problem of reducibility of the theory of algorithms, Dokl. Akad. Nauk SSSR 108 (1956), 194-197. (Russian)

4. John Myhill, Creative sets, Z. Math. Logik Grundlagen Math. 1 (1955), 97-108.

5. E. Post, Recursively enumerable sets of positive integers and their decision problems, Bull. Amer. Math. Soc. 50 (1944), 284-316.

Cornell University 